\title{
Super-resolution in near-field acoustic time reversal using reverberated elastic waves in skull-shaped antenna
}

\author{
Michael Reinwald ${ }^{1)}$, Quentin Grimal ${ }^{1)}$, Stefan Catheline ${ }^{2)}$, Lapo Boschi ${ }^{3)}$ \\ 1) Sorbonne Université, CNRS, INSERM, Laboratoire d'Imagerie Biomédicale, \\ LIB, F-75006, Paris, France. michael.reinwald@upmc.fr \\ 2) LabTAU, INSERM, Centre Léon Bérard, Université Lyon 1, Univ Lyon, F-69003, Lyon, France. \\ 3) Sorbonne Université, CNRS-INSU, Institut des Sciences de la Terre Paris, \\ ISTeP UMR 7193, F-75005, Paris, France.
}

\section{Summary}

We investigate the potential of using elastic waves for near-field acoustic time reversal, and in doing so evaluate the possibility of reconstructing sound source positions at below-wavelength distances from a skullshaped acoustic antenna. Our work is based on a conceptual processing model that translates elastic waves conducted and reverberated in an elastic object into source position, through a time reversal analysis. Signals are recorded by passive sensors glued on a replica of a human skull, measuring solely its mechanical vibrations, and not sensitive to airborne sound. The sound source is placed along the azimuthal and sagittal planes for distances to the skull between 5 and $100 \mathrm{~cm}$. We reconstruct the source position for signals with frequencies in the physiological hearing range with a resolution indirectly proportional to the distance between source and skull across all measurements in the far-field. Measurements in the near-field show $-3 \mathrm{~dB}$ widths smaller than half a wavelength (super-resolution) with highest resolutions of down to $\lambda / 15$ measured in front of the orbital cavities. We infer that these anatomical details give rise to complex features of the skull's Green's function, that in turn enhance resolution in a direction-dependent manner.

\section{Introduction}

It is well known [1] that anatomy contributes to the task of auditory source localization, as its effects on an acoustic signal, described by the head-related transfer function (HRTF) [37, 21], can be seen as a spectral filter and depend on the location of the signal's source. Human auditory source localization mostly relies on differences in the phase and amplitude of signals perceived by the two ears, as well as "spectral cues", or frequency-dependent effects associated with the shape of the pinnae and, possibly, other features of the body $([34])$.

Building on the work of Catheline et al. [7], we ex- plore here the specific role of elastic waves mediated in a skull-shaped object mimicking bone-conducted sound. While this study does not address the issue of whether and how bone conducted sound is employed by the human auditory (ears/brain) system, our goal is to determine whether these reverberated signals contain specific information about the reconstruction of the position of an auditory source, especially in the near-field. This could be relevant to current efforts in the study of bone conduction sound $[36,35,31,25,32]$. Using the principle of acoustic time reversal $[16,18]$, we convert the signal recorded by two receivers into the spatial coordinates of a source in the horizontal and sagittal plane, and evaluate the resolution with which the source position is thus reconstructed.

Catheline et al. [7] showed via a time reversal experiment with a dry skull that in-skull elastic wave propagation provides information about spatial positioning of a sound source. They found that their time reversal algorithm, using elastic waves alone, received at two or only one recording transducer mimicking the ear, successfully reconstructed the source position(s), for single as well as multiple sources. The spatial resolution of this method was found to decrease with increasing distance between the skull and the sound source. This is in good agreement with the far-field diffraction law, which provides a relationship between the spatial resolution and the distance separating the antenna (skull) from the source. Our objective is to expand the early work of Catheline et al. [7] and Ing et al. [22] to (1) analyze the resolution of the same algorithm for a skull-shaped antenna specifically in the near-field, i.e., the sound source is placed closer than one wavelength to the skull, and (2) to evaluate the directionality of the algorithm, i.e. evaluate changes in resolution with respect to angular position of the sound source.

In this study, we conduct a suite of experiments on a simple setup, equivalent to the setup used in Catheline et al. [7], consisting of two recording transducers glued to a replica human skull. Sound is generated 
by a small speaker deployed at a variety of distances and azimuths. Our results show in particular that, in the near-field, the resolution with which we reconstruct the source position changes as a function of azimuth with respect to the skull and is clearly influenced by complex features of the skull such as the orbital cavities. Furthermore we achieve superresolution throughout all angles for sources very close to the skull.

Similarly minded experiment have been conducted in recent years e.g. in the context of optics, where imaging with evanescent waves allows to surpass the classical diffraction limit; the super-resolution of nearfield microscopes is piloted by their probe size [28, 24]. In this context, a source [20, 12, 4] or scatterers [14] smaller than a wavelength, placed within the medium can be detected in the far-field with super-resolution as well. Time reversal experiments can also surpass the diffraction limit when resonators are placed near a source $[23,29]$ or when an acoustic sink is used [9]. To a lesser degree, near-field details can sometimes be extracted from the far-field using sophisticated algorithms such as inverse filter [8] or MUSIC [30]. Experiments with metamaterials, superlenses and hyper-lenses [27] demonstrate moderate sub-diffraction imaging down to a quarter of the optical wavelength. All these techniques use different terminology but they all require some near-field measurements.

Because very few studies in psychoacoustics have explored human sound localization performances for nearby sources [26], we are unable to determine whether the resolution achieved by our algorithm reproduces the performance of human listeners using bone conducted sound. While we do find that elastic waves contain sufficient information to successfully reconstruct source positions in the near-field, we cannot yet establish whether a similar capability is achieved by the human auditory system.

\section{Methods}

The experimental setup is based on the previously conducted experiment of Catheline et al. [7]: We use a skull-shaped object (for simplicity from now on called skull) made of the epoxy resin. The skull is mounted on a rotatable rod with a reference (horizontal) plane chosen approximately as a plane passing through the area of the ethmoid bone above the vomer and through the zygomatic arch and process of the temporal bone. A conventional loudspeaker (RS Pro TRG040008) is deployed sequentially at a discrete set of positions in the horizontal and vertical plane. The loudspeaker shows a flat frequency response between $200 \mathrm{~Hz}$ and $8 \mathrm{kHz}$. The distance between the source (loudspeaker) and the skull (the point on the surface of the skull closest to the speaker), denoted $D$, varies from 5 to $100 \mathrm{~cm}$, while the source position at each distance varies with angle $\varphi$ between $-50^{\circ}$ (i.e. down,left) and $+50^{\circ}$ (i.e. up, right). The experiment is conducted in an anechoic chamber. Equipment which could possibly reflect sound is covered with multiple layers of sound dampening material. Two passive sen-

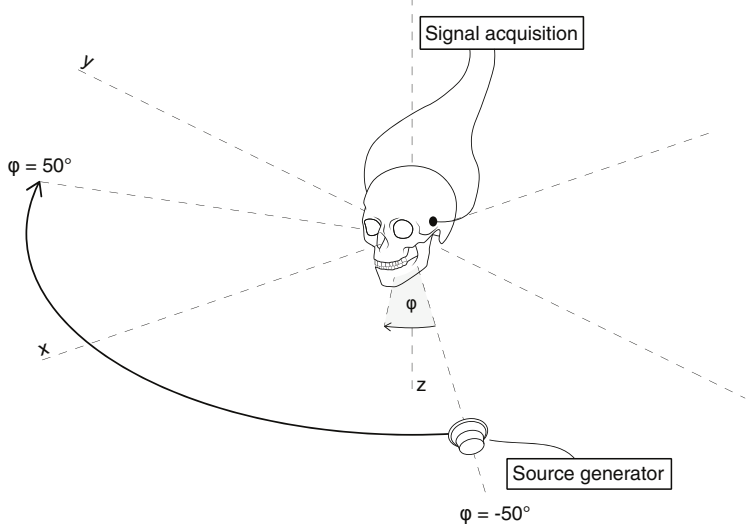

Figure 1: Sketch of the experimental setup in the horizontal plane: A loudspeaker is connected to a source generator (PC) and emits a chirp signal at each angle $\varphi$ ranging from $-50^{\circ}$ to $50^{\circ}$ along a half circle at various distances to the skull. The resulting vibration of the skull is recorded through two passive sensors glued to the hypothetical ear locations. They are connected to the signal acquisition system, consisting of a sound card connected to a PC.

sors (Murata PKS1-4A), with a working bandwidth ranging between $100 \mathrm{~Hz}$ and $15 \mathrm{kHz}$ and a diameter of $1 \mathrm{~cm}$, are glued close to the hypothetical ear locations on both sides of the skull. They are used as receivers to record the elastic vibrations and are connected to a sound card (Soundscape SS8IO-3) which has a $140 \mathrm{~dB}$ dynamic range and a $44.1 \mathrm{kHz}$ sampling frequency.

A sketch of the experimental setup in the horizontal plane is shown in Figure 1.

We checked that the sensors solely measure the vibration of the skull and are unresponsive to airborne sound. This ensures that the time reversal algorithm will utilize only elastic waves. Additionally, the influence of the foam platform used to place the loudspeaker at certain distances has been tested to have no influence on sound emission of the loudspeaker.

The first part of the experiment consists of recording the signals at the sensors for each speaker position. The speaker emits a chirp signal $c(t)$ with a duration of $1 \mathrm{~s}$ and a linear frequency distribution between $0 \mathrm{~Hz}$ and $6 \mathrm{kHz}$. The function in time for such a chirp of duration $T$, minimum frequency $f_{0}$ and maximum frequency $f_{1}$ reads

$$
c(t)=\sin \left[\Phi_{0}+2 \pi\left(f_{0} t+\frac{k}{2} t^{2}\right)\right],
$$

with the initial phase $\Phi_{0}$ at time $t=0$ and the chirpy- 
ness $k=\frac{f_{1}-f_{0}}{T}$ (in our case $k=6000 \mathrm{~Hz} / \mathrm{s}$ ), also known as the rate of frequency range across the chirp. For each distance $D$ the source positions in the horizontal plane are defined by the azimuth $\varphi$.

The recorded signal $s$ at one of the sensors' location $r$, writes

$$
s\left(\varphi_{0}, r, t\right)=c(t) * G\left(\varphi_{0}, r, t\right),
$$

where $*$ denotes convolution, $\varphi_{0}$ is the source position (azimuth) and $G\left(\varphi_{0}, r, t\right)$ is the acoustic impulse response of the skull, which is also the Green's function of the signal emitted at $\varphi_{0}$ and recorded at $r$, assuming without loss of generality that emitter and receiver are punctual. A representative waveform of a signal recorded with one of the sensors and its normalized frequency spectrum is shown in Figure 2. Note that

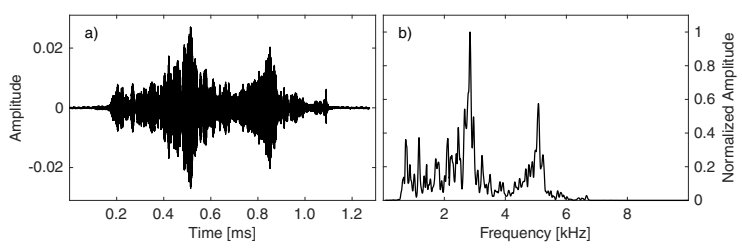

Figure 2: a) Exemplary waveform of a recorded signal at one of the sensors. b) Frequency spectrum of the same signal.

the spectra of all impulse responses (only one shown here) show strong similarity to the results from Catheline et al. [7] where a real dry skull was used and its resonance frequencies were confirmed with other studies of dry skulls and cadaver heads $[32,19]$. This proves that, in the first approximation and for the purposes of our study, the epoxy skull replica employed here is sufficiently similar to a real skull. It should be noted that, firstly, epoxy can have mechanical properties similar to those of bone tissue $([2,3])$; secondly, the most important role in our experiments is presumably played by the outer shape of the skull, driving wave propagation in air around the skull: and the replica is designed to have realistic external shape.

Following Fink [17], the received signal $s\left(\varphi_{0}, r,-t\right)$ is time-reversed, i.e flipped with respect to time. It must then be backward propagated to any possible location $\varphi_{i}$. This is equivalent to convolving $s\left(\varphi_{0}, r,-t\right)$ with the Green's function $G\left(\varphi_{i}, r, t\right)$. Since we do not have access to $G\left(\varphi_{i}, r, t\right)$, but we do have a library of recordings of $s\left(\varphi_{i}, r, t\right)$ for all possible values of $\varphi_{i}$, we implement

$$
\begin{aligned}
& T_{i}\left(\varphi_{0}, r, t\right)=s\left(\varphi_{0}, r,-t\right) * s\left(\varphi_{i}, r, t\right)= \\
= & c(-t) * G\left(\varphi_{0}, r,-t\right) * c(t) * G\left(\varphi_{i}, r, t\right) .
\end{aligned}
$$

The term $G\left(\varphi_{0}, r, t\right) * G\left(\varphi_{i}, r,-t\right)$ is the transfer function of such a time reversal algorithm and, in terms of signal analysis, represents a matched filter [17]. This convolution coincides with the cross-correlation of $G\left(\varphi_{0}, r, t\right)$ and $G\left(\varphi_{i}, r, t\right)([13,11])$. For each source position $\varphi_{0}$, the signal processing procedure consists of implementing Equation 3, i.e. analytically crosscorrelating the signals, and of finding the maximum value, with respect to time, of the time-reversed wave field $T_{i}$ for each $\varphi_{i}$. The resulting function $F\left(\varphi_{i}\right)$ is dubbed "spatial focusing function" (shortly, focusing function), as this procedure is equivalent to evaluating whether (and with what resolution) the timereversed and backward-propagated wave field is able to reconstruct the original source position $\varphi_{0}$. The focusing function is next normalized with respect to its maximum; It is then reasonable to assume that, the closer $F\left(\varphi_{i}\right)$ is to 1 (i.e., identical Green's functions) for a given value of $\varphi_{i}$, the closer $\varphi_{i}$ is to the original source $\varphi_{0}$. This method can be interpreted as a pattern recognition system, that identifies, from an acoustic reference library, the Green's function corresponding to the actual position of the source, and so determines the position of the source.

The invariance under time reversal is lost if the propagation medium has frequency-dependent attenuation. This introduces a first-order time derivative in the governing propagation equation. However, the theorem of spatial reciprocity is still valid, i.e. there is a loss of amplitude in the time-reversed vs. forward propagating wave field, but this does not affect source-localization resolution (does not affect the location of the focus of the time-reversed wave field) provided that signal-to-noise ratio of recorded data is sufficiently high. We have accordingly chosen to carry out our experiments at frequencies that are well caught by our receiving system.

We take both sensors into account by computing the mean of the focusing functions of the two signals.

In order to investigate the role of different frequency contents, the originally measured signals are successively filtered with varying low-pass filters with maximum frequency $f_{\max }$.

Following e.g. $[22,5,33]$, we estimate the spatial resolution of our time reversal algorithm by analyzing the $-3 \mathrm{~dB}$ width $\Delta p$ of $F\left(\varphi_{i}\right)$ for each given source position (angle $\varphi$ and distance $D$ between the source position and the skull) and various smallest wavelengths $\lambda_{\min }=c / f_{\max }$ (with $\mathrm{c}=$ speed of sound in air).

We compare our resolution estimates against the apparent aperture $A$ of our skull-shaped antenna, as defined by Catheline et al. [7], through the far-field diffraction law

$$
A=\frac{D \cdot \lambda_{\min }}{2 \Delta p} .
$$

While resolution as defined here is known to follow the diffraction-law in the far-field [7], that is not the case in the near-field, where Equation 4 is only used here for the sake of comparison. 


\section{Results}

\subsection{Verification of diffraction law}

In this section, we reproduce the results of Catheline et al. [7] and verify that our far-field data are consistent with the diffraction law (Equation 4 ). The source position is chosen to be at $\varphi=0^{\circ}$, which is in front of the center of the skull. We calculate the normalized focusing function $F\left(\varphi_{i}\right)$ along the curvilinear abscissa in the horizontal plane as described previously, for each distance to the skull. This is shown in Figure 3

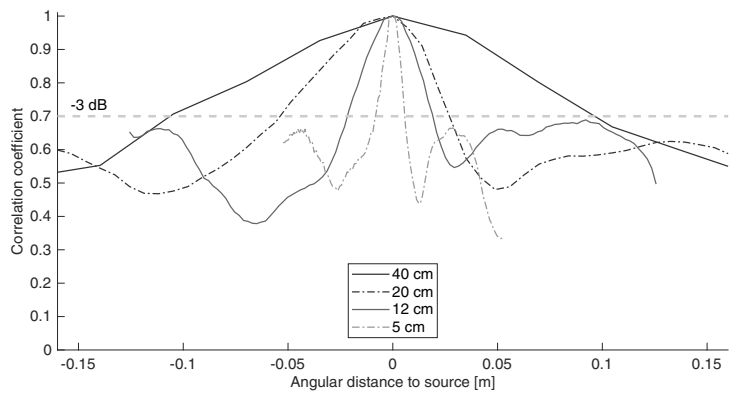

Figure 3: Normalized focusing functions along the curvilinear abscissa for sources in front of the center of the skull $\left(\varphi=0^{\circ}\right)$ and at different distances to the skull. The distance of the measurement points to the skull decreases from $40 \mathrm{~cm}$, down to $20 \mathrm{~cm}, 12 \mathrm{~cm}$ and $5 \mathrm{~cm}$ (different curves). There is a clear trend of increasing resolution (decreasing $-3 \mathrm{~dB}$ width of the curves) with decreasing distance.

as a function of the curvilinear abscissa. The $-3 \mathrm{~dB}$ (correlation coefficient of 0.7 ) widths of the curves are in good agreement with the diffraction law, confirming the findings of Catheline et al. [7], where the width of the curve is directly proportional to the distance between skull and sound source. Additionally it can be seen that the maximum peak to ground level (frequently named contrast) of our time reversal scheme lies below $-3 \mathrm{~dB}$. This has been confirmed for all measurements and ensures that calculating the resolution is not hindered by a low-contrast focusing function. Figure 4 shows the $-3 \mathrm{~dB}$ widths of the focusing functions of the signals for sources with different maximum frequencies $f_{\max }$ and at different distances in front of the skull $\left(\varphi=0^{\circ}\right)$. We calculate the values of $A$ using Equation 4 and the values shown in Figure 4 . They are found to be approximately $10 \mathrm{~cm}$ for all distances and maximum frequencies proving that the apparent aperture in the far-field is independent of distance or maximum frequency.

Measurements in the sagittal plane (not shown here) show smaller slopes of the linear fits evaluated in the same way as in Figure 4 across all results. Compared to the case of the horizontal plane, therefore the apparent aperture size is larger for these measurements $(15 \mathrm{~cm})$. This may be related to the different

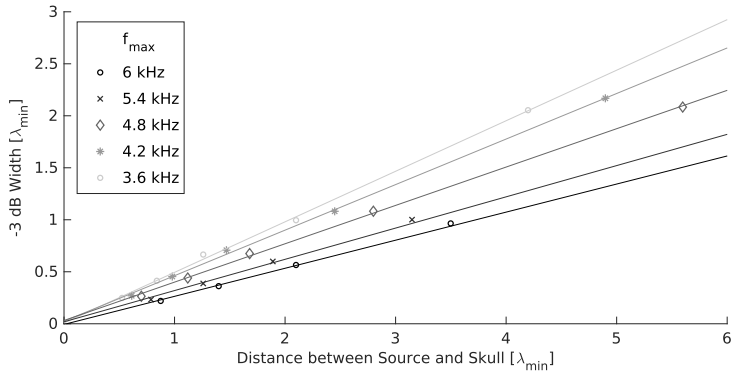

Figure 4: $-3 \mathrm{~dB}$ width values of the focusing functions for sources at different distances to the skull (x-axis) and maximum frequencies $f_{\max }$ of the signal. The slope of each linear fit, which corresponds to the apparent aperture $A$ in Equation 4, is approximately $10 \mathrm{~cm}$ for all curves.

diameters of the skull, close to 10 and $15 \mathrm{~cm}$, in the horizontal and sagittal planes, respectively.

The measurement points in the near-field (at distances smaller than one wavelength) lie on the same linear fit (i.e. same apparent aperture) as the points for measurements in the far-field although Equation 4 does not hold true in the near-field. In the near-field, i.e. for sources closer than one minimum wavelength away from the skull, source positions can still be resolved with the same angular resolution which results in super-resolution in space, i.e. $-3 \mathrm{~dB}$ widths below $0.5 \lambda_{\min }$ (see Figure 4 ). While one could infer that the diffraction limit also holds true in the near-field, our results are purely empirical; any values below the previously formulated diffraction limit are not represented in Equation 4. We speculate that they can be ascribed to the near-field contribution of evanescent waves.

Our far-field data is in agreement with Equation 4 and the previous findings of Catheline et al. [7]. In addition, we are able to achieve the same angular resolution as stated in the far-field diffraction law in the near-field (sound sources at below-wavelength distances) leading to super-resolution.

\subsection{Directional variation in resolution}

We furthermore investigate the directional variation of resolution of the time reversal analysis in the horizontal plane. The angular variations in resolution of our time reversal scheme in the near-field are visualized in Figure 5 showing the values of $A$ (top) and $\Delta p$ (bottom) with respect to the source azimuth $\varphi$ for different source distances $(5 \mathrm{~cm}, 12 \mathrm{~cm}$ and $20 \mathrm{~cm}$ and $100 \mathrm{~cm}$ ). All data is filtered to have a maximum frequency of $3 \mathrm{kHz}$. The reason for an offset of around $2-3^{\circ}$ to the center $\left(\varphi=0^{\circ}\right)$ is due to a limited accuracy in the manual placement of the center position and the center of the rotation axis.

In the far-field, the apparent aperture does not vary with azimuth (see $100 \mathrm{~cm}$ data in Figure 5) and is 


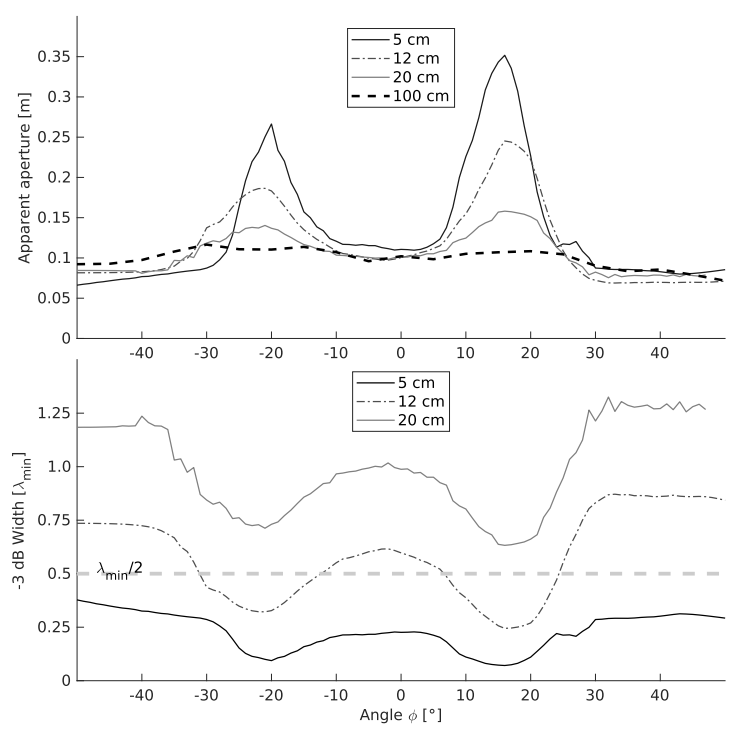

Figure 5: Angular variations of resolution for different source distances. Top: Variation in apparent aperture for different source distances. Maxima are at $-20^{\circ}$ and $15^{\circ}$ whereas the values decrease for source positions close to the center and further away from the center. Bottom: Variation in $-3 \mathrm{db}$ widths for different source distances. Super-resolution is accomplished throughout all angles at a distance of $5 \mathrm{~cm}$ and for certain angles at a distance of $12 \mathrm{~cm}$. Highest resolution (smallest $-3 \mathrm{~dB}$ width) is accomplished for source positions directly in front of the orbital cavities. This effect is (relatively) enhanced the closer the source to the skull.

equal to the value of $10 \mathrm{~cm}$ obtained from Figure 4 throughout all far-field measurements at source azimuth $\varphi=0^{\circ}$.

In the near-field, the largest apparent aperture values lie roughly in front of the two orbita, at $-20^{\circ}$ and $15^{\circ}$, and are up to more than three times larger compared to the aforementioned far-field value, whereas source positions in front of the nasal bone or along the process of the temporal bone show values closer to $10 \mathrm{~cm}$. The closer the source to the skull, the more prominent the angular directionality of the apparent aperture. Hence, the maximum apparent aperture is more than three times larger than the skull diameter in the horizontal plane.

$-3 \mathrm{~dB}$ widths are smaller than half a wavelength (super-resolution) throughout all azimuths at a distance between source and skull of $5 \mathrm{~cm}$, down to $\lambda_{\min } / 15$ (i.e. for $\varphi=-20^{\circ}$ and $15^{\circ}$ ). This shows that the skull-shaped antenna enables sub-wavelength focusing of near-field sources and, furthermore, anatomical details of the skull may give rise to differences in resolution at certain positions due to the presence of evanescent waves. They can be described as a nonpropagative spatial fluctuation field that decreases exponentially over roughly one wavelength [10] and can be created at a boundary between two media through certain incident angles of a propagating wave [15]. Usually, their effect is not measured in the far field and the far-field diffraction law (Equation 4) does not account for such effects, limiting the resolution of time reversal. However, if near-field components of the wavefield are measured and incorporated in the time-reversal algorithm, subwavelength information, that is carried by evanescent waves, is incorporated in the time-reversal process, leading to super resolution [23].

All these results are also approximately achieved via a one-sided evaluation of the signals, i.e. when only one receiver is used.

In summary, our data shows large variations in resolution in the near-field, depending on the position of the source relative to the geometric complexities of the skull.

\section{Conclusion}

In this study we measured elastic wave signals in a replica of a human skull due to an incident airborne sound emitted by a source at various distances and orientation with respect to the skull. Our goal was to investigate the physical limits of a sound-localization algorithm that uses full waveform information and the information contained in elastic waves propagating in the skull bone. While we do not at all claim to directly reproduce the sound localization "algorithm" that exists in the human ear-brain system, our quantification of these limits may be considered as a point of comparison in near-field psychoacoustics experiments.

We showed that the resolution of a time reversal scheme using a skull-shaped antenna with one or two receivers is consistent with the diffraction law in the far-field. The apparent apertures in the horizontal and sagittal planes are roughly consistent with the horizontal and vertical extent of the skull. Interestingly, the apparent aperture in the near-field is markedly increased (more than 3 times its value in the far-field) in the horizontal plane and at specific angles. In that case we can achieve super-resolution that may be associated to the non-negligible contribution of evanescent waves in the near-field.

Our results suggest that anatomical details of the skull give rise to complex features of the radiated sound field in the near-field, enabling sub-wavelength focusing and directional changes in resolution. We clearly find the influence of small anatomical geometric complexities such as the orbital cavities to positively influence resolution using elastic waves. We believe that it will be useful, in future studies, to explore the performance of our algorithms in other frequency ranges and for other biological models (e.g., echolocating species such as dolphins or bats).

As noted by Parseihian et al. [26], very few studies in psychoacoustics have explored human sound lo- 
calization performances for nearby sources (e.g. [6]). It appears to us that further experimental work is needed to more robustly evaluate how well humans localize nearby sources and if our findings can be related to psychoacoustic studies in the near-field.

\section{Acknowledgement}

This project has received funding from the European Union's Horizon 2020 research and innovation programme under the Marie Sklodowska-Curie grant agreement No 641943 (ITN WAVES).

\section{References}

[1] Arthur H Benade. Fundamentals of musical acoustics. Courier Corporation, 1990.

[2] Simon Bernard, Quentin Grimal, and Pascal Laugier. Resonant ultrasound spectroscopy for viscoelastic characterization of anisotropic attenuative solid materials. The Journal of the Acoustical Society of America, 135(5):2601-2613, 2014.

[3] Simon Bernard, Joannes Schneider, Peter Varga, Pascal Laugier, Kay Raum, and Quentin Grimal. Elasticity-density and viscoelasticity-density relationships at the tibia mid-diaphysis assessed from resonant ultrasound spectroscopy measurements. Biomechanics and modeling in mechanobiology, 15(1):97-109, 2016.

[4] Eric Betzig, Jonathan Trautman, Tim Harris, Joseph Weiner, and Robert Kostelak. Breaking the diffraction barrier: optical microscopy on a nanometric scale. Science, 251(5000):1468, 1991.

[5] Peter Blomgren, George Papanicolaou, and Hongkai Zhao. Super-resolution in time-reversal acoustics. The Journal of the Acoustical Society of America, 111(1):230-248, 2002.

[6] Douglas S Brungart, Nathaniel I Durlach, and William M Rabinowitz. Auditory localization of nearby sources. ii. localization of a broadband source. The Journal of the Acoustical Society of America, 106(4):1956-1968, 1999.

[7] Stefan Catheline, Mathias Fink, Nicolas Quieffin, and Ros Kiri Ing. Acoustic source localization model using in-skull reverberation and time reversal. Applied physics letters, 90(6):063902, 2007.

[8] Stephane G Conti, Philippe Roux, and William A Kuperman. Near-field time-reversal amplification. The Journal of the Acoustical Society of America, 121(6):3602-3606, 2007.

[9] Julien de Rosny and Mathias Fink. Overcoming the diffraction limit in wave physics using a time-reversal mirror and a novel acoustic sink. Physical review letters, 89(12):124301, 2002.

[10] Julien de Rosny and Mathias Fink. Focusing properties of near-field time reversal. Physical Review A, 76(6):065801, 2007.
[11] Arnaud Derode, Eric Larose, Mickael Tanter, Julien De Rosny, Arnaud Tourin, Michel Campillo, and Mathias Fink. Recovering the green's function from field-field correlations in an open scattering medium (1). The Journal of the Acoustical Society of America, 113(6):2973-2976, 2003.

[12] Robert M Dickson, Andrew B Cubitt, Roger Y Tsien, and William E Moerner. On/off blinking and switching behaviour of single molecules of green fluorescent protein. Nature, 388(6640):355-358, 1997.

[13] Carsten Draeger and Mathias Fink. One-channel time-reversal in chaotic cavities: Theoretical limits. The Journal of the Acoustical Society of America, 105(2):611-617, 1999.

[14] Claudia Errico, Juliette Pierre, Sophie Pezet, Yann Desailly, Zsolt Lenkei, and Olivier Couture. Ultrafast ultrasound localization microscopy for deep superresolution vascular imaging. Nature, 527(7579):499 $508,2015$.

[15] Mathias Fink. Time reversal of ultrasonic fields. i. basic principles. IEEE transactions on ultrasonics, ferroelectrics, and frequency control, 39(5):555-566, 1992.

[16] Mathias Fink. Time-reversed acoustics. Scientific American, 281(5):91-97, 1999.

[17] Mathias Fink. Acoustic Time-Reversal Mirrors. Imaging of Complex Media with Acoustic and Seismic Waves, 17:17-43, 2001.

[18] Mathias Fink. Time-reversal acoustics in complex environments. Geophysics, 71(4):SI151-SI164, 2006.

[19] Bo Håkansson, Anders Brandt, Peder Carlsson, and Anders Tjellström. Resonance frequencies of the human skull invivo. The Journal of the Acoustical Society of America, 95(3):1474-1481, 1994.

[20] Stefan W Hell and Jan Wichmann. Breaking the diffraction resolution limit by stimulated emission: stimulated-emission-depletion fluorescence microscopy. Optics letters, 19(11):780-782, 1994.

[21] Shichao Hu, Jorge Trevino, Cesar Salvador, Shuichi Sakamoto, Junfeng Li, and Yôiti Suzuki. A local representation of the head-related transfer function. The Journal of the Acoustical Society of America, 140(3):EL285-EL290, 2016.

[22] Ros Kiri Ing, Nicolas Quieffin, Stefan Catheline, and Mathias Fink. In solid localization of finger impacts using acoustic time-reversal process. Applied Physics Letters, 87(20):204104, 2005.

[23] Geoffroy Lerosey, Julien De Rosny, Arnaud Tourin, and Mathias Fink. Focusing beyond the diffraction limit with far-field time reversal. Science, 315(5815):1120-1122, 2007.

[24] Aaron Lewis, Michael Isaacson, Alec Harootunian, and A Muray. Development of a 500 å spatial resolution light microscope: I. light is efficiently transmitted through $\lambda / 16$ diameter apertures. Ultramicroscopy, 13(3):227-231, 1984.

[25] Tom Littler, John J Knight, and PH Strange. Hearing by bone conduction and the use of bone-conduction hearing aids. Proceedings of the Royal Society of Medicine, 45(11):783, 1952. 
[26] Gaëtan Parseihian, Christophe Jouffrais, and Brian FG Katz. Reaching nearby sources: comparison between real and virtual sound and visual targets. Frontiers in neuroscience, 8, 2014.

[27] John Brian Pendry. Negative refraction makes a perfect lens. Physical review letters, 85(18):3966, 2000.

[28] Dieter W Pohl, Winfried Denk, and Mark Lanz. Optical stethoscopy: Image recording with resolution $\lambda / 20$. Applied physics letters, 44(7):651-653, 1984.

[29] Matthieu Rupin, Stefan Catheline, and Philippe Roux. Super-resolution experiments on lamb waves using a single emitter. Applied Physics Letters, 106(2):024103, 2015.

[30] F Simonetti. Localization of pointlike scatterers in solids with subwavelength resolution. Applied physics letters, 89(9):094105, 2006.

[31] Stefan Stenfelt. Implantable Bone Conduction Hearing Aids, 71:10-21, 2011.

[32] Stefan Stenfelt and Richard L Goode. Boneconducted sound: physiological and clinical aspects. Otology \& Neurotology, 26(6):1245-1261, 2005.

[33] Chrysoula Tsogka and George C Papanicolaou. Time reversal through a solid-liquid interface and superresolution. Inverse problems, 18(6):1639, 2002.

[34] John Van Opstal. The auditory system and human sound-localization behavior. Academic Press, 2016.

[35] Bruce N Walker, Raymond M Stanley, Nandini Iyer, Brian D Simpson, and Douglas S Brungart. Evaluation of bone-conduction headsets for use in multitalker communication environments. Proceedings of the Human Factors and Ergonomics Society - 49th Annual Meeting, 49(17):1615-1619, 2005.

[36] Jack J Wazen, Jaclyn Spitzer, Soha N Ghossaini, Ashutrosh Kacker, and Anne Zschommler. Results of the bone-anchored hearing aid in unilateral hearing loss. The Laryngoscope, 111(6):955-958, 2001.

[37] Elizabeth M Wenzel, Marianne Arruda, Doris J Kistler, and Frederic L Wightman. Localization using nonindividualized head-related transfer functions. The Journal of the Acoustical Society of America, 94(1):111-123, 1993. 